\title{
Relating Maslow's Hierarchy of Needs with Employee Turnover and Retention: Case Study of Local Telco
}

\author{
Ayesha Hanif (Corresponding author) \\ Dept. of Engineering Management, CASE University \\ Islamabad, Pakistan \\ E-mail: ayeshah.hanif@gmail.com \\ Wajiha Khalid \\ Dept. of Engineering Management, CASE University \\ E-mail: wajiha.peracha@gmail.com \\ Dr. Tahir Nawaz Khan \\ Dept. of Engineering Management, CASE University \\ E-mail: tahirnawaz78692@yahoo.com
}

Accepted: May 19, 2013 Published: June 14, 2013

Doi:10.5296/ijhrs.v3i2.3864 URL: http://dx.doi.org/10.5296/ijhrs.v3i2.3864

\begin{abstract}
Purpose: In this case study, we investigate the frequency and reasons for the junior employees' turnover problems being faced by a growing Telco in Pakistan. Having identified the root causes, creative HR people practices have been suggested as solutions for retaining valuable talent.
\end{abstract}

Methodology: A survey questionnaire was administered among the staff of the company under study to identify their needs satisfaction level and then asked them to rate factors instrumental for retaining high performing talent. A Multi-point Rating Scale (1-5 and 1-7) was used for the 33 items.

Findings: The results explore which employee retention measures can be taken so as to improve employee job satisfaction, loyalty and productivity. The trend points along dimensions of financial benefits, improved work environment and growth opportunities.

Practical Implication: On broader platform, we can generalize the employee retention factors ranking to other Telco/IT companies operating in the hyper-inflated environment of Pakistan.

Value: In many local growing organizations, effort and finance is not invested to determine Employee Satisfaction Index on an annual basis. This study will serve the purpose of preliminary survey in this regard for this company in addition to providing insight into common Pakistani lower-cadre employee needs. 
Keywords: Employee Turnover, Employee Retention, Employee Satisfaction, Pakistan

\section{Introduction}

CompanyA has a functional structure. Despite global recession, the company made 100 new hirings, gave 150 promotions, distributed 370 individual awards and arranged 20 trainings in last year alone. The salary scale in NTRN can be quoted as a general trend starting from PKR $10 \mathrm{k}$ and having a raise of PKR $3 \mathrm{k}-4 \mathrm{k}$ raise per scale in addition to annual $10 \%$ raise whereas DepartmentA, engineers have a starting salary of 20-25k. In DepartmentB, the job description broadly comprises of in-house cabling, ducting and wiring, equipment installations at customer premises, pulling cable in underground ducts and working in tough weather conditions. For DepartmentA engineers, job description includes 24/7 support to customer queries, issuance, escalation, racking and resolution of trouble tickets.

The company offers several benefits to its employees, some of which are: Annual Bonus (with greater percentage for junior staff than for seniors), Health insurance, Provident fund, Extended leaves in case of on-the-job injury, Loan policy for motorbike purchase, Family fund for marriage-based needs, Annual awards for 6-7 NTRN employees worth Rs.10k, Employee of the Quarter award worth Rs.7.5k distributed quarterly, Best customer service and excellence awards worth Rs.5k distributed monthly, Overtime payment on 1.5-2X times of gross salary, Mobile phone facility to Supervisors and above, etc.

Promotions are based on excellent past performance, interview and recommendation by respective managers. Promotion period is dependent upon any expansion in the existing or new functions, completion of one year since joining/last promotion and performance feedback by manager on completion of assigned targets.

\section{Profiling of CompanyA through STAR Model}

\subsection{Strategy}

Over the years, the strategy of expanding services and building trust of the customers through quality of services has been the punch line at CompanyA. Organization also invested heavily in ensuring qualified line and managerial staff to support their strategy.

This strategy has helped the organization in building a sustainable customer base which has resulted in ever growing revenues and repute in comparison to its competitors.

\subsection{Structure}

CompanyA has functional structure with different departments performing their specialized jobs under their defined reporting channels. Different departments have different levels of hierarchy (span of control from two to eight tiers) and progression opportunities depending upon growing operational needs in a particular department in conformance with organizational needs. The decisions are inspired through top management (Centralization); however, day-to-day operations are conducted under respective management. This compartmentalization has helped in effectively utilizing skills of different cadres and contributed in achieving organizational objectives as enunciated by the management based on continual eye over the business requirements. 


\subsection{People Practices}

CompanyA works on hire-train-fire paradigm. Recruitment is made through exposing candidates to a range of technical, personality and psychological tests. The belief of the management in hiring the best from the market has ensured quality services delivered to the customers.

After recruitment, organization invests in improving skills of the technical staff through in-house trainings before they are given independent assignments in the field. Due to functional compartmentalization of the organization, people seldom find chance for rotations from one department to another.

It has resulted in limited growth initiative left for the employees who find it extremely disturbing to have touched the ceiling of growth within a period of 12-18 months after induction into the cadres of the organization.

\subsection{Processes}

CompanyA has a defined set of vertical and lateral processes. Top management provides the necessary guidance which is translated into action through middle management acting through their line staff. Cross functional coordination is carried out through respective department head who shares / seeks advice, where required. Cross Functional Teams (CFTs) for planning are formed as desired by the management; they are of temporary nature and routine matters are sorted out internally within respective departments.

There is strong lateral communication within departments with regular feedback mechanism which ensures eradication of faults through coordinated team work.

Informal culture is encouraged across teams that helps in building rapport at intra and inter team levels. It helps keeping the environment congenial for work.

\subsection{Rewards}

Company salaries are below market line for first-line and middle managers as well as for benchmarked jobs of field technicians which doesn't look healthy in comparison to salary structures in regional gulf market. Recognition programs are run for rewarding outstanding employees.

\section{The Challenges Encountered}

In DepartmentB, about 40 or so DAEs/non-DAEs belonging to AES, ES and ATE posts mostly have left in past one year. In DepartmentA, 40 new employees were hired whereas 26 departed from the company after an average service period of 20 months. The reason most often quoted in employees' exit interviews is the enticing pay package and brand name offered by recruiting companies in the Middle East. The company's take on this plight is mild, if not cold. They have accepted that it's no less than a bandwagon-effect and no matter how much pay raise and benefits they offer, the field technicians working in DepartmentB will head off to Saudi Arabia and Dubai. The hiring and training of these employees is costly and when skilled employee's turnover is in such large numbers, it should sound warning signs to the management and HR. 
The top management and HR planners are not feeling too much pressure due to no dearth of fresh technicians at lower induction level. Training cost compared to weighing budgeted cost of salaries for experienced staff cannot be justified with efforts to seeking advantage of trained staff through infusing loyalty.

\section{Literature Review}

In the time before 1980s, loyalty to the company meant lifetime of employment in that corporation. Now the younger lot has seen some disturbing examples like massive layoffs, closing pension funds etc. happening to their elders and so they are eager to hop jobs for more lucrative opportunity, no matter how short-term they maybe Error! Reference source not found.. "People in their twenties have a different view of work-life balance, and companies are going to have to engage them more and be more accommodating." According to Herzberg's theory, employees are motivated by work content (motivators) and work environment (hygiene factors). Therefore by investing in the education and development of its people, just like any other economic investment, an organization can gain a sustainable competitive edge and increase its market capitalization. This investment will create engaged workforce; a workforce that's enthusiastic and fully involved in its work. Unengaged employees add to absenteeism and poor quality work. It has been quoted that, "Climate is very much like the collective states of mind of all the people in any given organization. It can even be referred to as the level of mental health of an organization."

Researchers have examined the psychological as well as economic and environmental implications of an organization but few studies have taken into account the cultural aspect of a society contributing to employee turnover Error! Reference source not found.. This study has supported in finding three cultural dimensions namely personal characteristics, job satisfaction, and alternative employment opportunities adopted from Hofstede's model by analyzing their effects on employee turnover in different public sector, private sector and multinational organizations of Australia and China. China and Australia are believed to be running under two different cultural thoughts. While Chinese believe in valuing collectivism, longer future term goals and higher future uncertainty avoidance attributing to large power distance society, Australian people are believed to value individualism, self-fulfillment, self-satisfaction owing to less power distance in society and they are more incentive driven. Frequent job hopping was observed among people in Australia, while Chinese do not go for alternative employment opportunity more often due to cultural impact. On the basis of these three cultural dimensions, five hypotheses were formulated to test the subjects under study. Various tests were conducted in this regard including ANOVA, t-test to test significance in group results, correlation and regression to test the presence of either negative or positive relationship between test variables. Study results proved the presence of higher levels of job satisfaction in Chinese employed personnel as compared to Australian people and this supported hypothesis 1 rating Australian people higher on turnover ratios, disproved hypothesis 2 further strengthening the above stated argument that the more satisfied people are with their current job, the less they would go for a job hop. Results also proved that Chinese employees would most likely to go for a longer career prospect while Australian's would go for short term work incentive in performing their jobs. 
Error! Reference source not found.Explores relationship among work values, organizational identification and employee turnover intentions in the service sector firms of china. In the modern era where organizations worldwide are rapidly shifting towards service sector, there is an ever increasing demand of planning and adopting strategies regarding retaining the best talent. Therefore, it is the responsibilities of Human Resource Management to identify factors which can help an organization retain its employee over a longer period of time. The framework adopted is based on finding the effect/relationship of work values on organizational identification (OI) and employee turnover and finding the effect of organizational identification on employee turnover. OI can be taken as a key predictor between employees and organization in determining turnover intention. OI can be attributed to factors like self-categorization and self-conception which help an employee assess his work contentment. The more satisfied a person is with his current job, the less likely he would be to opt alternative employment opportunities. Work values identified for this particular research were intrinsic value of self-motivation, extrinsic reward value, promotional value and social status value. Intrinsic value is found to moderate the relationship between OI and employee turnover positively. Whereas the other three work values are found to moderate the relationship negatively. Correlation and regression results concluded that employee turnover intentions are dominated by reward values and social status values. Social status was found out to be one moderating variable which positively affected both OI and employee turnover.

Error! Reference source not found. Takes into account Chinese manufacturing sector and studies the level of employee commitment and the factors that help employees to stay in their current organizations. Nowadays, organizations are aggressively trying to keep ahead of their rivals and to do so retaining their best human talent is the first thing HR department tries to do. Authors in this paper refer to employees as the core competency of any organization, and stress upon the fact that if such employees are not provided suitable incentives like adequate participation, suitable job environment etc. they are most likely to leave the job for a better opportunity. A considerable amount of resources are also required on recruiting and training newly hired personnel, so organizations prefer retaining their best employees rather than bringing in talent from outside which is not so familiar with organization's culture and norms. Research construct takes into account 8 dimensions affecting employee commitment for any organization namely the nature of job itself, interpersonal relations, enterprise condition, communication, leader elements, participation in management, promotion and development and fairness in the organization. Results showed that employee turnover rate varied significantly with the change in demographic factors like age, gender, marital status, education etc. Out of the 8 dimensions described in [4], 5 were found to account for nearly $62 \%$ of employee commitment alone. Employee turnover was found to be nearly $35 \%$ dominated by employee commitment. Results from the factor analysis and expert grading showed that the greater contributors of employee commitment are job itself, interpersonal relations, participation in management, fairness in organization and leader elements.

It's generally considered that greater the job satisfaction and organizational commitment, lower is the employee turnover rate. However these factors are termed as controllable factors because they lie within the circle of organization's control. There should be an optimal 
employee turnover rate i.e. the out flux and influx of employees should be balanced, and it should neither be too low that it causes stagnation, nor so high that organization is always on the verge of losing valuable employees. This relationship between employee turnover rate and organizational performance can be expressed as a down U-shaped curve. Recruiting right people for the right job and retaining them over a long period of time would ensure that the company gains and sustains its competitive advantage and grows concurrently. Incentives like competitive salaries, job trainings and development programs come under the umbrella of controllable factors and can be instrumental in employees attaining higher levels of job satisfaction and organizational commitment. To a certain level, uncontrollable factors can be minimized by providing attractive packages, ample training and development opportunities.

Various researches have tried to prove an exact relationship between organizational performance and employee turnover, but no exact relationship has been deduced yet Error! Reference source not found.. Research carried out till now has worked out four possible relationships between organizational performance and employee turnover and they are positive relationship, negative relationship, no significant relationship, or contingent relationship among the two variables. Those researchers who emphasize the existence of no significant relationship between the two variables argue that an employee's absence may be considered as a dip for a short time and may not significantly impact performance of an organization. Authors who are in favor of positive relationship between the two variables argue that poor performers are most likely to leave than good performers in most cases, so this may seem to have a negative effect in short term, but in longer term it is actually benefitting the organization that poor performers are naturally getting filtered. Most authors however argue that employee turnover indicates a loss to the organization; the costs associated with employees leaving the organization are quite high when it comes to recruiting, training and developing them. Also it represents loss in the form of losing valuable human resources especially if senior managers resign. It also takes some time for the new employees to raise their performance level to the expected level. High turnover may also cause a firm's performance to degrade rapidly. High conformance firms are not affected that much by employee turnover, however low conformance firms suffer performance degradation to a great level. Researchers who argue in favor of contingent relationship between the two variables state the relationship between the two in the form of a downward U-shaped curve. The curve shows that whenever turnover rate falls below $10 \%$ both the variables have a positive relationship, and when the turnover rate goes $20 \%$ above, the two variables have a negative relationship. In a nutshell, researches still haven't been able to conclude upon any one single relationship existence between the two variables.

The dysfunctional and controllable turnover should be avoided. Error! Reference source not found. Builds on the premises that organizations get feedback from employees regarding their opinions and attitudes towards the company but do not have a plan chalked out for how they will utilize the collected data for calculating why employees leave or stay. It is important because, "Sometimes it's not who you hire that counts; it's who you keep", be it young, middle-aged or top performers. It is said that a good manager is an avid student of cause and effect; he has to be aware of what is happening around him and understand why it is 
happening. Then he has to roll up his sleeves and get down to finding a solution. Once issues are identified, the matter should be escalated to management for appropriate actions. Employees should be able to see that their feedback makes a difference.

A critical question arises: When should retention efforts begin? Error! Reference source not

found. Says that it could start at the time of HR planning, recruiting, selecting and the commencement of the HR management. It has been established that the current market is a corporate culture centering on knowledge with knowledge playing the role to a company as blood does to a person. "The nuclear capability of the high-tech enterprises lies in the innovation mechanism and ability." Literature has introduced the term "Intellectual Employees' and author is of the view that company gains value in terms of creativity and innovation due to the intellect of these employees. 3\% of these IE's are the key members that should he retained at any cost, $13 \%$ are important staff that should be retained at any reasonable cost whereas $68 \%$ should be retained and be satisfied with their demands. IE's are also less loyal to the company because they get better job offers from competitors and develop an individualistic view. Therefore outflow of such employees is unaffordable and HR should have different strategies to discover, train and retain each category. 411 subjects doing marketing, administrative and technical work were surveyed using a questionnaire to appraise their contentment. It was found that males were more eager to flow out than females. And among them, those below 35 years of age, with bachelor's degree rather than masters, at supervisor or middle-level rather than top-level, technical staff and those with more than 3 years of experience have higher intensity of outflowing. The IE's demanded the following in descending order of importance: good salary, promotion and development, interesting and challenging job, fair judgment of one's work. The first demand is that of economic reward because to them, it is as standard of measuring their own value in the market. As per feedback received, the company surveyed should restructure its salary packages, pay more attention to training and development needs of employees and side-by-side the corporation is required to make a mental bond with the employees by making some commitments. The technical IE's can be given reasonable and impartial made-to-individual salary program. Valuable staff can be awarded share options to form a tie with the company. They absorb more and have a higher need for learning so training and a clear career design is essential. Provide them self-evaluation methods, less restricting supervision and flexible work timings.

Error! Reference source not found. Tells us that popular theories on motivation in North American literature were given by Vroom (1964), McClelland (1961), and Maslow (1943). Maslow's work inspired many motivation theories for e.g. expectancy theories, achievement motive and hierarchy of human needs. Maslow's theory has been validated in many cultures, including American culture in which it can be mapped on their society's high individualism, low uncertainty avoidance and high masculinity.

A nexus between Maslow's Hierarchy of Needs Framework and the Chinese practice of Guanxi is found in Error! Reference source not found.. Guanxi literally means "gateway to belongingness" and essentially is a social relationship setup in Chinese society. In Guanxi behavior, people give each other gifts, do favors and offer help to family, friends and people to whom they are connected interpersonally so that they can gain favors and meet personal objectives. This Guanxi practice can be used in organizational context as well. In the context 
of organizations, the lowest level physiological needs require proper working environment and financial compensation for the employees. Through Guanxi, people find a job which leads to fulfillment of their physiological needs. At the second level of safety and security, employees require fringe benefits, insurance and health plans etc. Through Guanxi practice, managers can help their subordinates cope with their emotional needs. Belongingness needs at workplace form the level three needs which can be fulfilled by forming working relationships with supervisors, managers and peers. This satisfaction of need for social ties is similar to Guanxi which is regarded as an "informal organization". The employees form relations with 'Shuren' who are recognizable colleagues with whom other people also have social relations. 'Renquing', doing favors, also helps form ties. Some companies hold luncheons, sports, common rooms, cross training etc. for this purpose. Stepping onto the fourth rung of the needs ladder, self-esteem is required by employees to feel respected and recognized. This can be correlated to the Chinese concept of 'face'. This is the reputation and respectability a person is regarded with in society. Many companies in Chine have honor societies and prestigious awards ceremonies to boost self-esteem of their employees. At the highest needs level of self-actualization, employee realizes the satisfaction of using his full potential and skills. Satisfactions of these needs of employees by managers lead to employees' motivation and productivity. However Guanxi comes with some risks. These include chances of nepotism, favoritism and loss of fairness when low-performing employees get rewarded only because of their skill at Guanxi.

Error! Reference source not found. Unearths the dichotomy of whether predictors that decide employees' intent to leave also depict his intention to stay? The implication that same factors influence both choices requires empirical testing. This probe can be legitimized in context of the Heisenberg's Theory too which says that improving the hygiene factors cannot increase job motivation as that is dependent on motivators alone, but absence of hygiene factors can cause dissatisfaction. This study intends to differentiate between antecedents that influence decision to stay but not decision to leave (retainers) from those that influence decision to leave but not the decision to stay (dis-engagers). 416 hospitality employees in USA were surveyed to find whether perceived organization support (POS), perceived supervisor's support (PSS) and organizational commitment (OC) affect both sides of the same coin; employee' intention to remain with or leave the organization. It was found that employees' intention to leave decreases as POS and OC increases however not as PSS increases. That is, when POS increases due to supervisor's beneficial treatment to an employee, the employee doesn't necessarily feel obligated to stay and help realize the organization's goals. When it came to employees' intention to stay, the effect of POS was twice as strong as that for the intention to leave. However, as PSS and OC increased, no influence was detected on intention to stay. So we can say that loyalty to the supervisor doesn't instate loyalty for the organization or positive feelings for one person in social network does not turn into positive feelings for the whole group.

Can employees be retained through intrinsic and extrinsic motivators? Error! Reference source not found. Describes a study set in two public and private organizations in South Africa; a country where employees are consistently leaving for better jobs abroad. A rebuttal is required by the South African organizations in the form of new retention strategies to 
compete with the continuous poaching strategy of employees by multinationals. The study in Error! Reference source not found. answers this question using a sample of 145 participants' surveyed using questionnaire. Employee turnover is being caused for many a reasons encompassing lack of recognition, lack of interesting work, uncompetitive compensation program, non-conducive work environment etc. Using chi-square method to test hypotheses, it was found that training and development, job security, liberty to be innovative and challenging work were motivators for employee retention.

Companies often fear that if they train their employees, they will leave for better opportunities by banking on the newly acquired and polished skills. However, it was found in Error! Reference source not found. that trained people have higher retention percentage than untrained ones. The retention stats were studied for $3000+$ employees in a study. The employees could attend training anytime within 6 months of joining the firm. In the 3-day training session, called Transitions, trainees were motivated to build a successful career at the company. Moreover the participants assessed themselves as to where they stood in after leaving their previous company and having joined the new one. Experienced hires who attended the Transitions and who did not attend were compared on basis of four benchmarks using ANOVA and survival analysis techniques. It was found that this course attendance had significant impact on how long the employee stayed at the organization. Benchmark 1 (retention percentage at a point in time) indicated a $4 \%$ higher retention value for those who attended the Transitions. On Benchmark 2 (retention rate over time), those who received training had a $95 \%$ retention rate in the first year which dropped to $80 \%$ in the second year. For the untrained hires, the retention rate was $80 \%$ in both years. Another benchmark for comparison is the length of time required to reach a particular retention percentage, which was chosen as $80 \%$ in this case. The group that attended training reached this benchmark after 550 days in comparison to 300 days value for those who weren't among the recipient of Transitions training. The most important of the benchmarks from management point of view is the amount of revenue raised by the trained people to compare with the cost invested in their training. The tuition, on-site and air travel expenses proved to be minimal as compared to revenue generated by trained employees as it was $\$ 12$ raised for every $\$ 1$ invested. So training not only helps in retention but also improves performance of trainees.

We have formed the opinion in Error! Reference source not found. that training helps retain employees but it has also been proved that as a secondary advantage, training also helps retain the mentor. One such example exists at The Michigan Tech where two programs, GUIDE (Graduate and Undergraduate Initiative for Development and Enhancement) and ExSEL (Excelling in Science and Engineering Learning) have been running successfully Error! Reference source not found.. These programs use peer mentoring to help retain females and other underrepresented minority students in engineering. The program was formed when the institute faced problems on first-year retention, specifically of females. It rummaged through many initiatives including outreach efforts, partnerships and retaining through research associations until it received a National Science Foundation Grant. Every year, the first-year students had second-year and graduate students as mentors. If these first-year students achieved good academic results, next year they got the chance to mentor fresh students. This setting has helped the underprepared students in their belonging needs 
and retained them in engineering field. The success is reflected in students' GPA rise, greater research participation, internships and involvement in more campus activities. The undergraduate mentors reported that through this exercise, their listening skills became sharp. The mentees reported that the training helped the most in adjusting to the college life. Additionally, the program achieved its true purpose of retention also by reporting $87 \%$ participation and a mentor retention rate of $97 \%$ over a 5 year period.

The practices are not just theoretical but have been implemented in many organizations around the world. For example, Tata Consulting Services (TCS) in India offer its employees the choice of working in over 170 offices across 40 countries Error! Reference source not found. Interestingly, they have found a way to link social service with employee benefits for e.g. a paternity leave if employee adopts a girl child. They also offer discounts on group parties. These measures have significantly reduced job hopping. At INFOFSYS, social activities for employees and their families are used in addition to wellness facilities and creating a corporate university to minimize job hopping. If we look at Mahindra \& Co. uses 'reverse mentoring' program in which young people are given opportunities to train senior employees. The result was stabilized job hopping.

SAS is a software development company counted among the Top 100 companies to work for in USA Error! Reference source not found.. They have provision for an unlimited sick leave for their employees, be it for six days or six months, with the belief that "if you treat people like adults, they will behave as adults." They also offer meals for employees' children in the company cafeteria for monthly charges of $\$ 250$. Employees are encouraged to lunch with their family. Healthcare is provided on-site ranging from full medical, dental and vision to hearing. The on-site facility helps the company save $\$ 300,000$ per year in health insurance costs and time is saved. Employees are encouraged to not stay at office past $5 \mathrm{pm}$ and the work week is 35 hours. If new employees are hired and have to be paid more, all existing employees of same skill level are given equal pay too.

At My Maid cleaning service, employees who stay with the company for two years get the opportunity to be trained in the career of their choice. This measure dropped their turnover rate from $300 \%$ in 2007 to zero in 2009 Error! Reference source not found.

Wegmans' is famous for its comprehensive grocery specialties, good pricing and customer service that serve as a cherry on top. It has made onto the list of Fortune Magazine's "100 Best Companies to Look for" for 7 years in a row. The company was able to achieve this with its satisfied workforce of 32000 , out of which $75 \%$ current managers have been with the company since they were 15-16 years old. 11000 of its employees have worked here for 5 years, 5400 have worked for 10 years and 6000 employees have their family members working at Wegmans'. Wegmans' have policy of providing flexible timings to students and academic scholarship program who work part-time or full-time at the company. Part-timers who are children of permanent workers at Wegmans' get the highest scholarship. They hold employee appreciation days on which the department managers fire the grill and cook for their employees.

Accenture is a consulting firm and jobs require extensive travelling. The management therefore works on the principle that success and retention is delivered if employees are offered flexibility in how they conduct business by balancing work and personal life. The 
flexibility is choses as suitable to employees up to partners and includes part-time, job-sharing, telecommuting, flex-time, flex leave, working at office table from 9-5 or from the couch at home etc. Around 225 employees in the age group of 55+ work through this flexible work arrangement. This way, the problem of baby-boomers retirement leaving a void behind is also catered.

Hunter Douglas, manufacturer of custom window coverings, uses mentoring, workplace satisfaction and communication to retain employees. The management realized that when an employee joins new workplace, it's a stressful time for him. To make this time comfortable for newly hired employees, they have a mentoring program. The program uses long-term employees to meet with these new employees at least once every week for the first 90 days. Supervisors learn to speak the dominant language of the workplace, like Spanish and Vietnamese, to assist new employees in understanding and adjusting with workplace mechanics and responsibilities.

The Society for Human Resource Management (SHRM) recognized Analytical Graphics as the best company to work for in USA. The company has some very creative practices to keep workforce engaged. There are on-site dry-cleaning and workout facilities in addition to free meals for employees and their family members. Employees are welcome to bring their children for whom there are laptops and Internet facility available to do homework and playroom to play. On Friday as the week wraps up, all employees get together for lunch including the CEO and off-site workers for 'story time'. The CEO starts by talking about his week stories, which could be personal or work-related. Then other people can join in. In this 60-90 minutes session the entire workforce connects in a way that no amount of written communication or memos can accomplish. These perks result in increased productivity. The Director HR sums the philosophy as, "You're a professional. We trust your judgment, or we wouldn't have hired you. This is where we want you to go [your objectives to reach], and you use your judgment to make that happen."

If we look at HR practices in the Sub-Continent, Tata Consulting Services offers its employees a choice of working in over 170 offices across 40 countries in a variety of areas. It also offers paternity leave if an employee adopts a girl child. This practice is creative because they are contributing to society along with employee retention. Similarly in WIPRO, under the 'Wings Within' program, employees are given the option of leaving their job and working in any other firm within WIPRO. At Mahindra and Co., they have institutionalized a practice called 'reverse mentoring' in which young people are given opportunity to mentor their seniors. In this way, seniors remain up-to-date with developments and technology and juniors get confidence.

When we compare HR practices in Pakistan with that of Western or other Asian nations, we observe that a rethinking and redesigning is required. At the same time, we also observe through the above examples that loyalty can't be bought by benefits and perks alone. It requires creation of a workplace where employees know they are cared for, invested in, trusted and treated with respect.

\section{Methodology}

\subsection{Participants}


We surveyed employees in CompanyA who worked in two departments comprising of field technicians and customer support respectively. The pay scale ranges between PKR 20k- 25k

(200-2500 USD) per month. The purpose was to find out which employee needs get satisfied within this salary and benefits in addition to enquiring what such employees expect from CompanyA to be satisfied employees such that they do not hop jobs.

\subsection{Tools}

The survey forms were developed after careful literature review on Maslow's hierarchy of needs, employee turnover and retention; with emphasis on Pakistani culture. 16 items in the questionnaire measured employee needs satisfaction level and on next 15-items, employees ranked the steps management can take to retain them. For demographic data, we asked for age and gender of employee, department and years of service at CompanyA.

\subsection{Method}

Questionnaires were floated by hand among employees in the two departments of CompanyA. It allowed for us to interact with the respondents. Additionally, the filled survey forms were collected back from the employees without the involvement of respective managers to ensure unbiased feedback. The questionnaire comprised of two parts. The first part defined on a Likert scale of 7 from 1=Never, 2=Rarely, 3=Occasionally, 4=Sometimes, 5=Frequently, $6=$ Usually to $7=$ Every time, assessed the current need satisfaction level of employees. The second part comprised of questions defined on a 5-point Likert scale ranging from 1=Not a priority, 2=Low priority, 3=Medium priority, 4=High priority to 5=Essential, which helped to assess the needs which employees would want to be taken care of by their organization.

The reliability analysis provided following results with $\alpha=0.842$ for the first questionnaire and 0.909 for the second questionnaire:-

\begin{tabular}{|r|r|}
\multicolumn{2}{c}{ Reliability Statistics } \\
\hline Cronbach's Alpha & N of Items \\
\hline .842 & \\
\hline
\end{tabular}




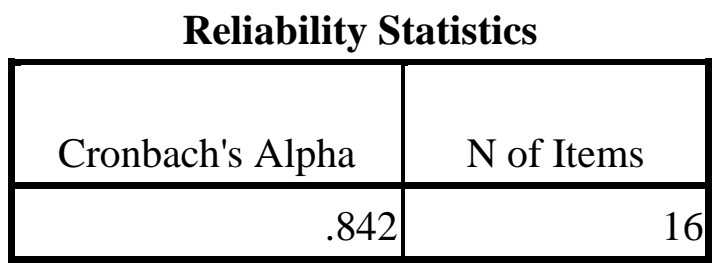

\section{Results}

Comparing the mean item values for both questionnaires, we were able to draw results by statistically evaluating them as shown in the SPSS results below. Descriptive statistics for the first questionnaire show a mean value of 4.17 for the physiological needs level, 4.00 for the security, 5.41 for belongingness, 5.5 for self-esteem and 4.56 for self-actualization needs level. From this it can be inferred that employees' needs for belongingness and self-esteem are frequently to usually fulfilled in the company they are working whereas they are only sometimes satisfied with their current physiological and security needs fulfillment.

\section{Statistics}

\begin{tabular}{|r|r|r|r|r|r|}
\hline & $\begin{array}{c}\text { physiologi } \\
\text { cal }\end{array}$ & security & $\begin{array}{c}\text { ingnes } \\
\mathrm{s}\end{array}$ & $\begin{array}{c}\text { self-este } \\
\text { em }\end{array}$ & $\begin{array}{c}\text { self-actuali } \\
\text { zation }\end{array}$ \\
\hline $\mathrm{N} \quad$ Valid & 36 & 36 & 36 & 36 & 36 \\
Missi & 0 & 0 & 0 & 0 & 0 \\
ng & & & & & \\
Mean & 4.1759 & 4.0093 & 5.4167 & 5.5208 & 4.5694 \\
Std. Deviation & 1.29342 & 1.10550 & 1.3282 & 1.03575 & .97945 \\
& & & 6 & & \\
\hline
\end{tabular}

For the

questionnaire, the mean results are shown below. Descriptive statistics show the mean value of 3.7 against the physiological needs level, 3.69 against security level, 3.75 for belongingness, 3.4074 for self-esteem and 3.57 for self-actualization needs level. This indicates their concern for improving their physiological, security and belonging needs by rating their opinions high on these three scales. Mean figures show that employees have placed "medium priority" for self-esteem and self-actualization needs and placed an emphasis on fulfilling their lower level needs by their respective organization. 


\section{Statistics}

\begin{tabular}{|c|r|r|r|r|r|}
\hline & $\begin{array}{r}\text { physiologica } \\
1\end{array}$ & security & belongingness & Self-esteem & $\begin{array}{c}\text { Self-actualizatio } \\
\mathrm{n}\end{array}$ \\
\hline 1 Valid & 36 & 36 & 36 & 36 & 36 \\
Missing & 0 & 0 & 0 & 0 & 0 \\
Mean & 3.7083 & 3.6991 & 3.7500 & 3.4074 & 3.5741 \\
Std. & .89742 & .86265 & 1.03701 & 1.03926 & .87509 \\
Deviation & & & & & \\
\hline
\end{tabular}

Additionally, interesting results which reinforce the un-met physiological needs can be observed by looking at the frequency of responses to items in $2^{\text {nd }}$ questionnaire. Some results are:

Better pay to cover expenses of house rent, transport and medical allowances?

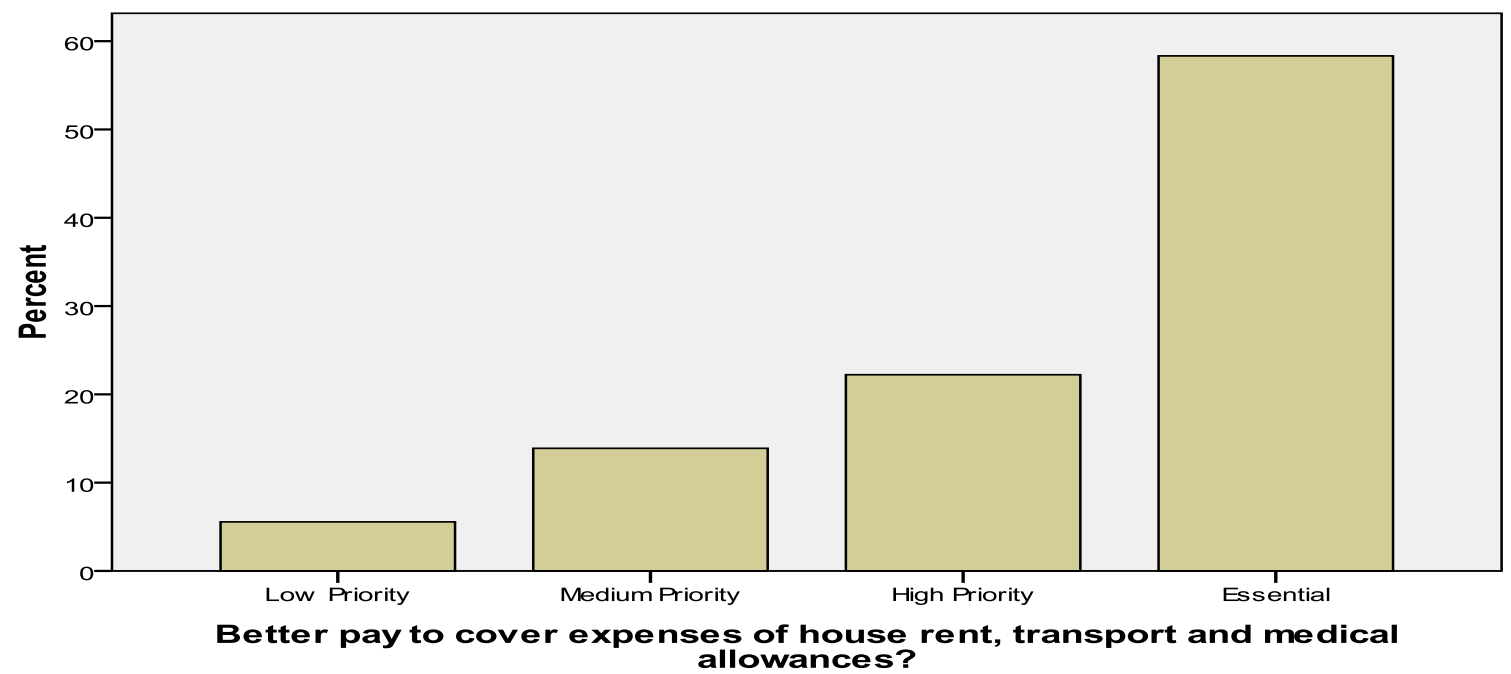

This is a very important result signifying that employees place their pay at highest priority of needs to be fulfilled. Company is not offering enough pay incentives to retain their current employees and this can be attributed as one major cause of their high turnover. To reduce the turnover rate CompanyA needs to revise pay plan and devise attractive pay packages to lure new employees and retain the present ones. 
Co-workers treating each other better?

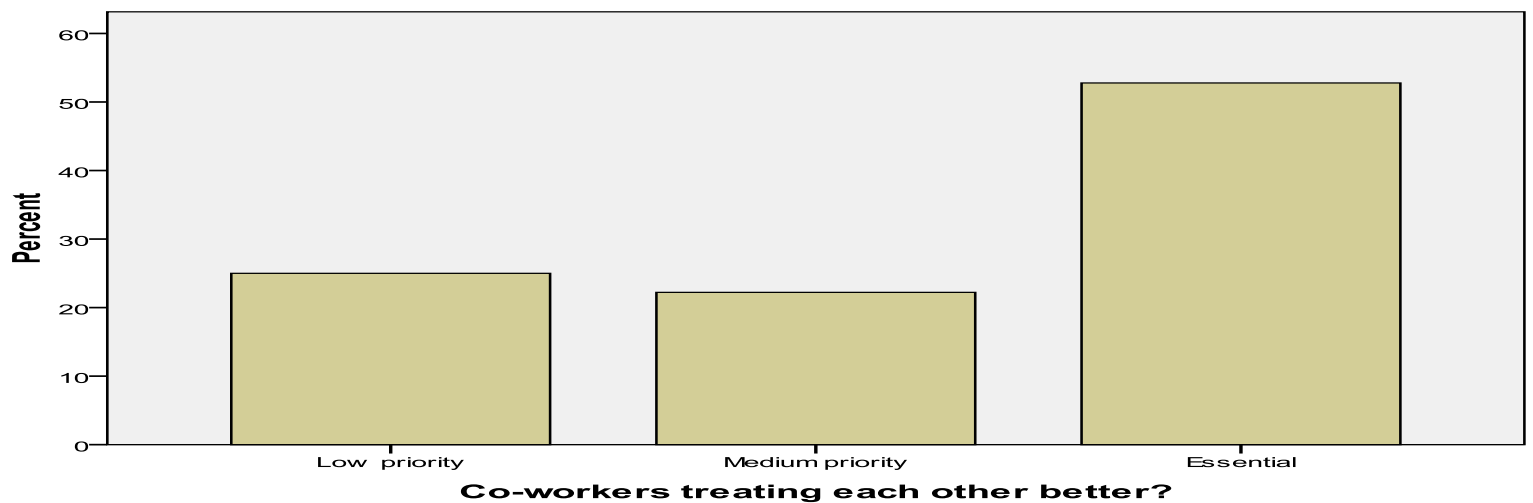

Result puts an emphasis on the presence of a good work environment where workers are essentially treating each other in a respectful manner. This is a great contributor in retaining employees over a longer period of time as it will satisfy their belonging needs. Following results also support this argument:

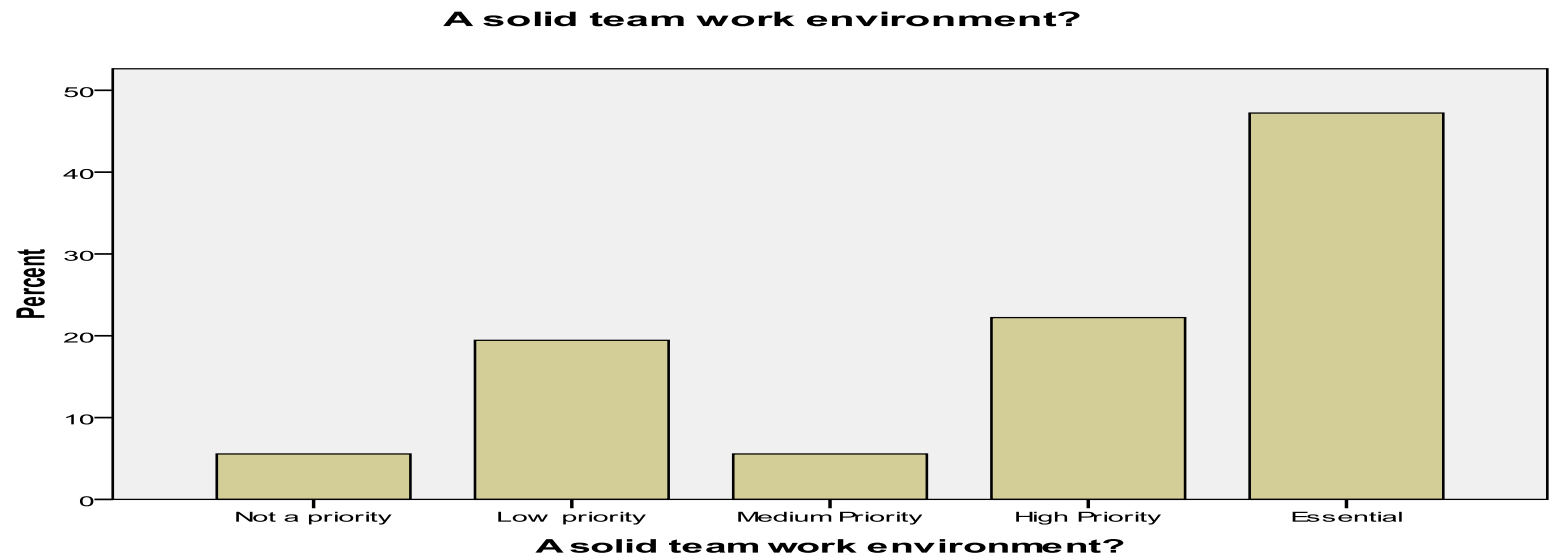

Rewards and benefits are emphasized by the employees:

Bonuses after training someone?

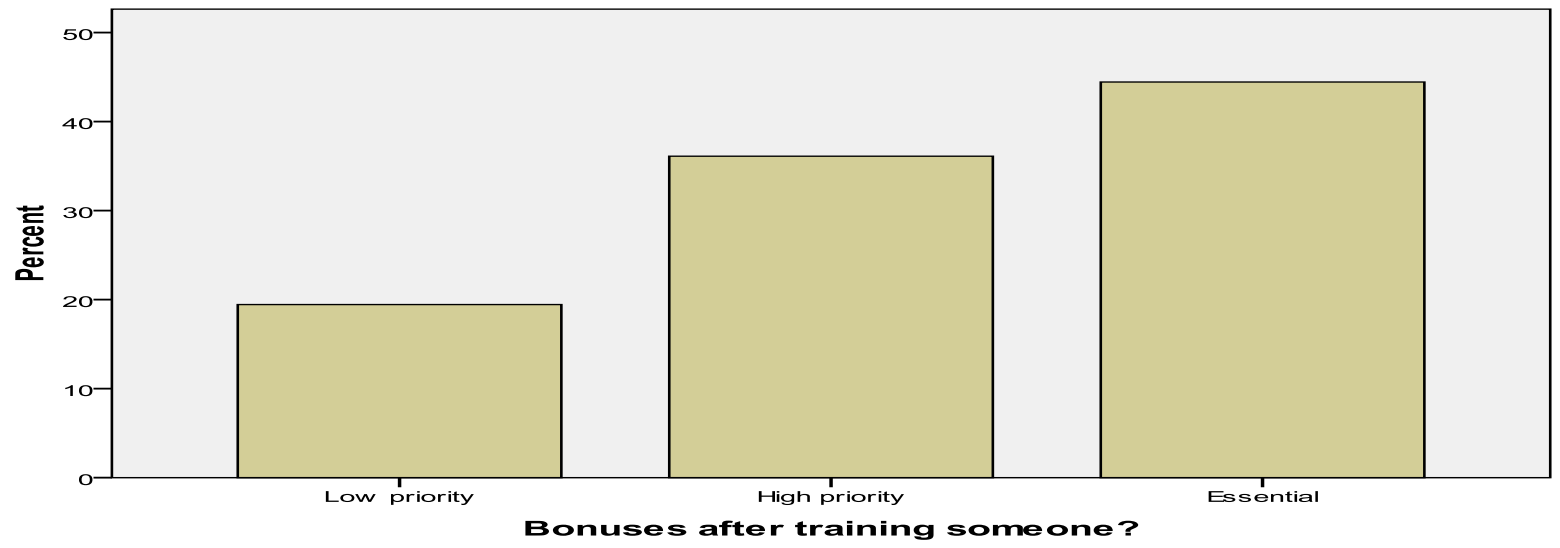

Consensus is also found on the need to expand skills and competencies: 


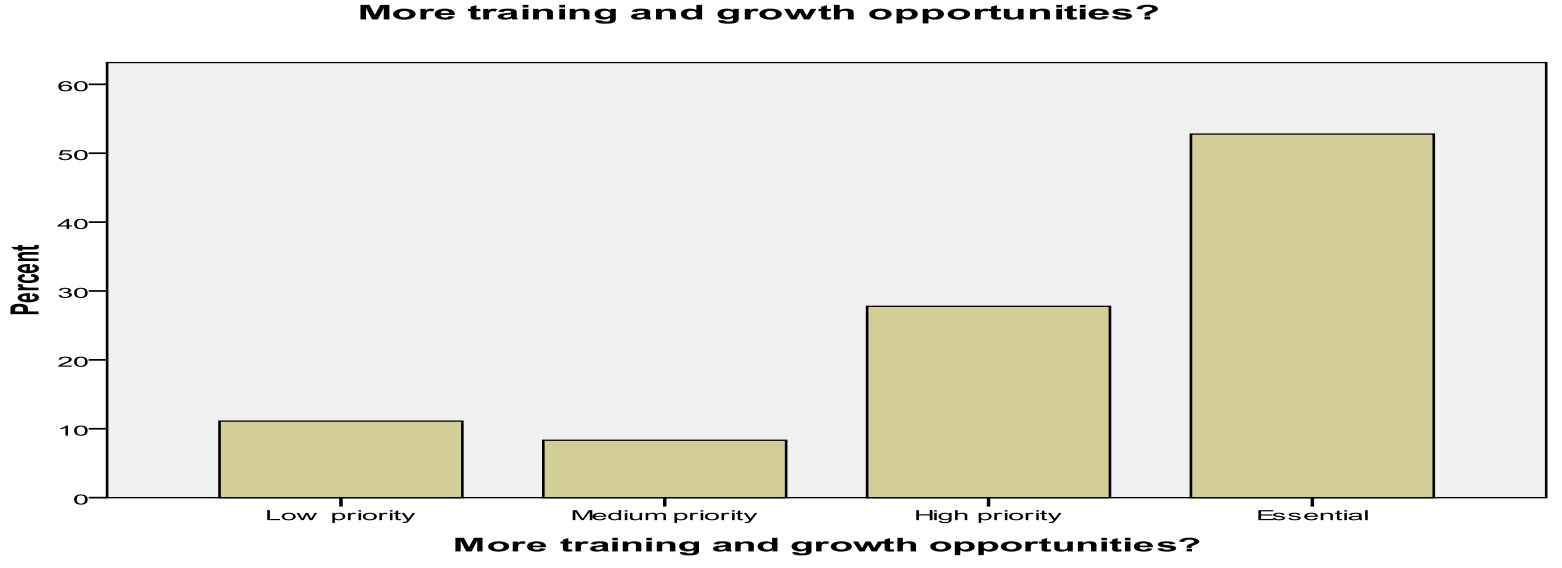

\section{Discussion}

Results show a contradiction with Maslow's hierarchy of needs assumptions that lower level needs should be satisfied first before moving up on the needs level. They also contradict with the underlying assumption that only one need can be catered at one time and multiple needs cannot be gratified at the same time. These two results are also the drawbacks of Maslow's hierarchy of needs which have been compensated for by other theories like the ERG theory, Herzberg's motivation theory etc. CompanyA needs to take care of pay incentives and monetary rewards in order to retain them over a longer term.

For non-supervisory staff and entry level employees, primary motivators are pay, food and shelter of more commonly the regular Cost-of-Living-Adjustments in salary. When employees take a fresh start with their careers, they aim to strengthen themselves financially and they would be prepared to leave their jobs for even a $10 \%$ salary increase. This poses problem with companies like CompanyA who offer low entry level salaries (PKR 20, 000) which caused the employees to rate themselves low on physiological and security needs. In DEPARTMENTB, as per calculation using Error! Reference source not found. turnover rate is $47 \%$, turnover cost per employee is Rs.50, 160 and annual turnover cost goes up to Rs.2, 006,400. The calculations have been made assuming the average length of each employee's job in as 1 year. Turnovers lead to hidden costs like new hiring's, trainings, a sharp decrease in organizational productivity during the new employee learning process, increased supervision required affecting other teams and employees and reduced levels of customer satisfaction.

Rapid promotions are also one way to retain field staff. There is $60 / 20 / 20$ rule which states that $20 \%$ of the people in your firms would like to be your partners, $20 \%$ would not like to be and $60 \%$ would like to be only if you have done all the things right. So, for those $60 \%$ doing the things right can spell the difference between admirable and non-admirable working places.

\section{Conclusion}

CompanyA prides itself in acquiring loyal customers. Retaining employees in which the company has invested is another side of the same coin! These employees, when happy with their jobs, can serve as the very best ambassadors for the company. If the company expects its 
employees to go the extra mile for its customers, it must prove that it is willing to go the extra mile for them by raising the pay percentile. Employee turnover in any organization should be maintained at an optimal level to ensure good progressing health of firm. Organizations that remain vital show their new employees that they are needed and create 'intrapreneurs'. At the same time, they never forget the value of their long-service employees.

\section{Limitations}

The scope of the study can be increased outside one organization to generalize the effects of results on the IT/Telecom sector. Also results can be evaluated against other motivational theories presented to get a vivid picture of how to respond to varying needs of employees by understanding the underlying reasons in a detailed manner.

\section{References}

[1] Yu hua, Xie, "Employee turnover and perception of incentive: A cross cultural comparison between China and Australia",Nov. 2010,pp.964-968

[2] Wei, Xueyan, "Chinese Employee's turnover intentions in relation to organizational identification, work values in modern service sector", June 2009, pp. 1-5

[3] Chen Yu, "Research on the Relationship between Employee Commitment and Turnover in the Equipment Manufacturing Industry of Shenyang", May 2011, pp. 1-4

[4] Wu, Zhigang, "Strategic Analysis of Employee Turnover", August 2011, pp. 1-4

[5] Changzheng, Zhang, "The relationship between employees' turnover rate and industrial firm performance: A literature review”, July 2010, pp. 383-387

[6] Employee retention and turnover: Using motivational variables as a panacea by Michael O. Samuel and Crispen Chipunza, African Journal of Business Management Vol.3 (8), pp. 410-415, September, 2009

[7] Understand Guanxi Practice in Chinese Organizations through Maslow's Hierarchy of Needs Theory by Bo Huang and Hua Xu 2012 International Conference on Information Management, Innovation Management and Industrial Engineering

[8] Using survival analysis to demonstrate the effects of training on employee retention by John R. Mattox II, Darryl L. Jinkerson Evaluation and Program Planning 28 (2005) 423-430, Elsevier

[9] Does Peer Mentoring Increase Retention Of The Mentor? By Amy E. Monte, Kerri A. Sleeman and Gretchen L. Hein IEEE Proceedings - Frontiers in Education Conference 11/2007; DOI:10.1109/FIE.2007.4417950

[10] A strategy to assist management in workforce engagement and employee retention in the high tech engineering environment by Elizabeth Kennedy, Tugrul U. Daim Evaluation and Program Planning Journal

[11] Cross-Cultural Perspectives on Motivation by Chulwon Kim USA Annals of Tourism Research, Vol. 25, No. 4, pp. 202 \pm 000 , 1998, Elsevier Science Ltd.

[12] Employees' intent to leave: A comparison of determinants of intent to leave versus intent to stay by Seonghee Cho, Misty M. Johanson, Priyanko Guchait International Journal of Hospitality Management 
[13] Flow and Retention of Intellectual Employees in Hi-tech Companies by CHEN Jin and WANG Li-yingIEMC '03, Managing Technologically Driven Organizations: The Human Side of Innovation and Change, Pages $176-180$.

[14] Human Resource Management in India: 'Where From' and 'Where To?' by Samir R. Chatterjee, Research and Practice in Human Resource Management, Volume 15, Issue 2, Pages 92-103

[15] Best Practices in Recruitment and Retention, ISBN 1-55645-134-2 http://www.attcnetwork.org/explore/priorityareas/wfd/grow/documents/Best\%20Pract ices\%20in\%20Recuitment\%20and\%20Retention.pdf

[16] Turnover Cost Calculation: http://www.expresspros.com/turnover/ 